\title{
Délie ou le recueil entre l'ordre et le sens
}

Xavier Bonnier

\section{Citer ce document / Cite this document :}

Bonnier Xavier. Délie ou le recueil entre l'ordre et le sens. In: Réforme, Humanisme, Renaissance, $\mathrm{n}^{\circ} 62, \mathbf{2}^{2006 .} \mathrm{pp} .73-$ 97;

https://www.persee.fr/doc/rhren_1771-1347_2006_num_62_1_3268

Fichier pdf généré le 29/03/2019 


\title{
Délie ou le recueil entre l'ordre et le sens
}

\author{
... uixque ipse reuerti \\ ad limen potuit; tanta est fallacia tecti \\ Ovide, Métamorphoses, VIII, 167-168
}

Jamais définitivement clos, sans doute, ne sera le débat sur la structure effective de la Délie de Maurice Scève. Si tous les critiques s'accordent à trouver quelque singularité dans l'inclusion régulière de cinquante emblèmes - tous les neuf dizains depuis l'intervalle entre le cinquième et le sixième jusqu'à celui qui sépare le D 446 de l'antépénultième - comme dans l'impeccable reconduction de la forme « carrée » de dizains décasyllabiques - hormis un huitain initial en 1544, qui sera final en 1564, et se situe de toute façon hors numérotation -, si tous également admettent que la réputation de savant de l'auteur, versé dans la science des chiffres comme dans l'architecture, et concluant son Microcosme par un tercet détaché qui insiste bizarrement ${ }^{1}$ sur la tripartition de l'œuvre en "trois mille, et trois vers », doit être prise en compte pour l'interprétation de son canzoniere, si tous enfin notent que le goût conjoint qu'il cultive pour le secret et l'ambiguiité - depuis l'anonymat jusqu'aux devises énigmatiques, « souffrir non souffrir », « non si non la » - fait qu'il paraît retirer d'une main ce qu'il propose de l'autre comme clé pertinente pour ses œuvres, aucun n'a pu, jusqu'à présent, fournir une grille de lecture absolument satisfaisante (au sens pragmatique d'indiscutable) et démêler l'écheveau de ces indications hétéroclites. C'est qu'au-delà de la pertinence des repérages numériques, donc d'une pure question d'ordre, se pose obstinément une autre question, plus redoutable parce qu'elle l'englobe sans en dépendre obligatoirement, venant ainsi la parasiter, celle du sens de l'œuvre.

Trois cas de figure sont en effet à considérer: ou bien la Délie a un sens global, c'est-à-dire présente une leçon bien claire et cohérente à partir (ou à propos) d'une expérience de passion amoureuse, et ce sens global est étroite-

1. Voir l'analyse, marquée par un salubre scepticisme, que lui consacre E. Giudici dans son édition du Mircocosme, Paris, Vrin, s.d. [1976], p. 467-471. 
ment lié - fût-ce par contraste, par tacite antiphrase - à un certain ordre déductible de l'architecture apparente du recueil; ou bien ce sens global existe, mais n'a que peu à voir avec l'ordre, et serait inchangé si, par exemple, Scève n'avait pas inclus d'emblèmes, ou avait opté pour une série de cinq cents dizains, ou de quatre cents sonnets, ou de quatre cent quarante-neuf dizains hétérométriques, etc.; ou bien, enfin, la Délie n'a pas de sens global satisfaisant, n'offre aucune leçon d'ensemble susceptible de résister à toute interprétation de détail, et alors la question de l'ordre du recueil devient tout à fait secondaire, puisque la structure, pure géométrie arbitraire, se révèle impuissante à guider l'interprétation, si même elle n'agit pas comme palliatif, selon une certaine tradition médiévale ${ }^{2}$. À l'intérieur de ces trois grandes hypothèses, une foule de possibilités se dessinent, d'intérêt très inégal évidemment. Mais la question majeure restant de savoir si, en tant que poète, Scève a délibérément construit son recueil en fonction d'un sens général qu'il invite son lecteur à retrouver, il convient dans un premier temps de passer en revue les grandes structurations déjà proposées, puis, à partir du constat de leurs différentes limites, de poser en termes si possible plus pertinents la question de l'articulation de l'ordre et du sens, et enfin d'esquisser une solution générale à partir de deux grands types de parcours déterminés.

Il serait prétentieux et fastidieux d'examiner dans leur détail, et par ordre chronologique d'apparition, toutes les hypothèses déjà formulées sur un ordre significatif du recueil scévien ${ }^{3}$. Une typologie de ces grilles de lecture, cependant, est à la fois nécessaire, possible et plus fructueuse, parce qu'elle indiquera plus efficacement les problèmes rencontrés quant aux principes mêmes de l'interprétation. Mais, avant d'en exploiter la substance, il faut garder à l'esprit deux éléments d'ordre historique assez importants.

D'une part, la réception de la Délie au XVI e siècle, qu'elle se traduise par l'admiration ou la désapprobation, fait, certes, une place de choix à la difficulté $\mathrm{du}$ recueil, les admirateurs y reconnaissant un art aussi hautain que souverain, les pourfendeurs une maladresse, mais toujours au seul niveau du vers ou du poème, pas à celui de l'architecture générale. Comme si le problème d'une correspondance entre l'architecture globale et le contenu informatif n'avait pas à se poser, la critique d'alors concentre son attention sur une dispositio de petit périmètre, phrastique, syntaxique, lexicale. Ou du moins, si ceux qui en appe-

2. «Au Moyen Âge, on était fort éloigné d'exiger l'unité du sujet et la cohésion de la composition. On voyait même dans l'egressio, l'excessus, une élégance particulière. [...] Cependant, pour remplacer la technique de composition moderne, le Moyen Âge possédait [...] la composition fondée sur les chiffres. " (E. R. Curtius, La Littérature européenne et le Moyen Âge latin, Paris, P.U.F., 1956, t. II, p. 328-330).

3. Un rappel satisfaisant en a été récemment donné par C. Alduy dans Délie object de plus haulte vertu, édition critique par Eugène Parturier, introduction et bibliographie par Cécile Alduy, Paris, STFM, 2001, p. XII-XV. 
laient sarcastiquement à un « docteur » pour suppléer au déficient « lecteur » 4 visaient autre chose que la densité de l'énoncé au cœur du poème, ils se sont bien gardés de l'expliciter. Et les défenseurs de Scève, Sébillet en tête, confirment a contrario cet empire de l'unité minimale du recueil ${ }^{5}$. Il faut attendre la toute récente modernité, et en particulier les savants calculs d'A.-M. Schmidt, pour trouver une hypothèse interprétative liant le sens à la structure numérique. La conséquence est claire: entre la déduction hâtive que ce qui ne comptait pas pour ses contemporains ne comptait pas non plus pour Scève, et l'hypothèse hardie qu'il avait élaboré, dans un isolement prophétique, une structure si fine qu'il ne comptait que sur la postérité pour la dévoiler, il faut tout simplement admettre l'éventualité d'une indifférence de l'auteur à la conversion de l'arithmétique en arithmosophique ${ }^{6}$.

D'autre part, la notion même de recueil n'est pas porteuse des mêmes enjeux, à la Renaissance, selon qu'il s'agit de la réunion de pièces de différents auteurs, ou de l'œuvre d'un seul. Même si Scève y a participé, fait patent pour le Recueil de vers latins et vulgaires paru en 1536 à la suite de la mort du Dauphin François ${ }^{7}$, ou seulement possible pour les Fleurs de poésie française ${ }^{8}$, de tels ouvrages n'engagent pas la problématique de l'ordre et du sens avec la même acuité que lorsque, seul maître de sa composition, l'auteur n'agit ni sur commande, ni selon un « cahier des charges » générique, tonal, et plus globalement rhétorique. C'est bien plutôt de recueils comme le Canzoniere pétrarquien, ou, en France, L'Adolescence clementine, Les Amours de Cassandre ou Les Antiquités de Rome, qu'il faut rapprocher la Délie en termes d'architecture signifiante9. Dans

4. « Tes vers sont beaux \& bien luysants,/Graves \& pleins de majesté,/Mais pour leur haulteur moins plaisants:/Car certes la difficulté/Le grand plaisir en a osté./Brief ilz ne quierent un lecteur,/Mais la commune autorité/Dit qu'ilz requierent un docteur. ", écrit Charles Fontaine dans une épigramme «A Monsieur Maurice Sceve », La Fontaine d'amour, Lyon, Jean de Tournes, 1545, p. 145.

5. Trouvant que la Délie est un "Poème d'autant riche invention qui pour le jourd'hui se lise ", il déplore que l'envie ne cesse de le mordre «de ses aiguës dents de chien, et trouve à reprendre en ces tant doctes épigrammes la rudesse de beaucoup de mots nouveaus, sans lesquels toutefois l'énergie des choses contenues celée et moins exprimée, eût fait ignorer bonne part de la conception de l'auteur, laquelle avec tout cela demeure encore malaisée à en être extraite. " Art poétique français, ch. IV, dans Traités de poétique et de rhétorique de la Renaissance, éd. F. Goyet, Paris, Livre de Poche, p. 61-62.

6. Il y a, certes, une troisième possibilité, à mentionner par acquit de conscience: que Scève ait élaboré une structure significative cryptée à destination de happy few contemporains qui, par principe ou à cause de la nature même du contenu secret, se soient contraints au silence - comme leurs éventuels successeurs.

7. Recueil de vers latins \& vulgaires de Plusieurs Poëtes Françoys composés sur le trepas de feu monsieur le Daulphin, Lyon, F. Juste, 1536 [BNF Rés. Ye 2966]. Huit contributions de Scève.

8. Hecatomphile. Les Fleurs de poesie françoyse, éd. G. Defaux, Paris, STFM, 2002. Tous les contributeurs n'ont pas encore été identifiés.

9. Qu'il suffise, à grands traits et sans exclusive, de rappeler par exemple la structuration en diptyque du Canzoniere, de part et d'autre du traumatisme de la mort de Laure; la progression d'une écriture poétique de plus en plus autonome et indiquant finement ses propres mutations successives dans l'Adolescence; le passage d'un discours sur l'échec amoureux à un discours sur la narration de ce même échec dans Les Amours; la respiration tonale entre sonnets décasyllabiques, plutôt lyriques, et sonnets d'alexandrins, plutôt syllogistiques, dans les Antiquités. Voir respectivement - et encore une fois plus par souci d'illustrer la possibilité de grilles de lecture à l'échelle d'un recueil que pour 
ce cas, une autre conséquence s'impose: le fait de proposer au public un recueil structuré indépendamment de la célébration d'un événement et de conception strictement individuelle revient à suggérer un programme de lecture spécifique, auquel l'anonymat confère en outre un statut de résolution d'énigme.

Ces précisions prises en compte, quelles sont les grandes structurations proposées? Une ligne de partage peut être tracée entre deux options: la première privilégie un trajet, une progression semblable à un cheminement, unique ou répété; la seconde privilégie au contraire un réseau, une structure statique, synchronique ou inerte.

À l'intérieur de la première option se sont dessinées deux grandes constructions interprétatives: d'une part l'hypothèse d'un parcours linéaire, fût-il d'aspect « diariste », qui voit dans la Délie le compte rendu par l'Amantpoète d'une aventure amoureuse inaugurée par l'innamoramento et débouchant, après une suite d'épreuves, sur le dépassement du sentiment amoureux au profit d'un amour idéal ou spiritualisé - c'est encore de cette manière que certains dictionnaires tâchent de "résumer ", avec une prudence variable, la matière du recueil ${ }^{10}$. D'autre part celle $\mathrm{d}^{\prime}$ une structure cyclique, ou au moins en spirale, prenant notamment pour appui la rotation du cadre géométrique des emblèmes ${ }^{11}$.

La première hypothèse est bien sûr le terrain d'élection des tenants d'un néo-platonisme quasi militant, qui voient dans la Délie un itinéraire d'épuration progressive inspiré par le ficinisme alors fort en vogue. Et certes les arguments ne manquent pas pour faire valoir cette interprétation globale, depuis certaines reprises thématiques, comme l'allusion au mythe de l'androgyne ${ }^{12}$, la

y voir parole d'évangile - l'introduction de P. Blanc dans son édition du Canzoniere, Paris, Bordas, 1988; J.-M. Colard, « L’Écriture comme « passetemps » », Cahiers Textuel n 16, Paris, Paris-VII, 1997, p. 81-92; J.-Cl. Moisan, «L'Organisation des Amours de Cassandre», Études littéraires, vol. 4, n² 2, Québec, 1971, p. 175-186; M.-M. Fontaine, « Le Système des Antiquités de Du Bellay: l'alternance entre décasyllabes et alexandrins dans un recueil de sonnets ", Le Sonnet à la Renaissance: des origines au XVII ${ }^{e}$ siècle, Paris, Aux Amateurs de Livres, 1988, p. 67-81.

10. «Au simple lecteur n'est accessible que l'histoire, assez capricieuse, d'une évolution sentimentale qui part, comme chez Pétrarque, du jour fatal et bienheureux de la rencontre, et qui, à travers une succession d'incidents et d'anecdotes, malgré les hésitations et les rechutes, change peu à peu l'ardeur sensuelle d'un homme en une passion pure, idéale, pour s'achever sur un vœu d'immortalité poétique. » (Dictionnaire des lettres françaises. Le XVI e siècle, Paris, Fayard/LGF, 2001, art. "Scève », p. 1077).

11. La recherche sur la signification des emblèmes et leur lien avec le texte du recueil constituant un champ à part entière depuis plusieurs décennies, il ne saurait être question ici de discuter dans le détail les positions énoncées. Il faut simplement se souvenir $1^{\circ}$ ) que la Délie n'est pas un livre d'emblèmes, comparable par exemple aux Emblemata d'Alciat, mais constitue le tout premier cas, en France du moins, de recueil sporadiquement illustré; $2^{\circ}$ ) que les contraintes typographiques ont joué un rôle décisif dans la répartition précédemment décrite (voir E. M. Duval, « Articulation of the Délie: Emblems, Numbers, and the Book », Modern Language Review, LXXV, 1980, p. 65-75); 3) que les emblèmes, qu'ils jouent en soutien ou en contrepoint du texte proprement dit - et les deux sont avérés -, doivent impérativement être pris en compte pour toute interprétation générale de l'œuvre, ne serait-ce qu'à cause du doublet répétition/variation, régularité/hétérogénéité qui les caractérise tout autant que les dizains (voir F. Lecercle, «Du Phénix au Pot-au-feu: les emblèmes de Délie et la poétique de l’hétérogène ", Europe, LXIV, n691-692, 1986, p. 95-101).

12. Au D 271. 
mention de " $\mathrm{l}^{\prime}$ hermaphrodite, efficace amoureuse ${ }^{13}$ et de la « tressaincte, \& sage Dyotime » associant Délie à l'institutrice de Socrate dans le Banquet ${ }^{14}$, ou la louange d'une beauté qui mène à la vertu par l'intermédiaire de la grâce ${ }^{15}$, jusqu'à certains choix lexicaux rien moins qu'innocents (la possible anagramme de «L'Idée » affirmée par La Croix du Maine, ou, de façon plus tangible, le «Ciel, non Peripatetique » du D 444, venu tout droit de Speroni et qui semble écarter la physique aristotélicienne au profit de l'idéal platonicien christianisé), en passant par le fameux distinguo entre "Amour ardent, \& Cupido bandé »16, et, surtout, l'affirmation récurrente d'une quête du « hault bien » et de l'immortalité à partir de la contemplation de l'Aimée, de la mort à soi-même de $l^{\prime}$ Amant pour ne vivre qu'en $l^{\prime}$ Aimée $^{17}$, et de sa lutte pour renoncer à la seule possession charnelle, autant de valeurs qu'illustre bien, comme en raccourci, ce dizain consolateur (D 134) évoquant le privilège faussement avantageux dont jouit le mari de Délie:

Saincte Union povoit seule accomplir

L'intention, que sa loy nous donna,

Comme toy seule aussi debvois supplir

Au bien, qu'a deux elle mesme ordonna.

A luy \& Corps \& Foy abandonna:

A moy le Cœur, \& la chaste pensée.

Mais si sa part est ores dispensée

A recepvoir le bien, qu'Amour despart,

La mienne est mieulx en ce recompensée,

Que apres Amour, la Mort n'y aura part ${ }^{18}$.

13. Au D 435 .

14. Au D 439.

15. Au D 306. La progression est tout à fait explicite.

16. D 217.

17. Un exemple frappant, parmi beaucoup d'autres, d'inspiration néo-platonicienne sur ce thème précis: lorsqu'il lit «Celle beaulté, qui embellit le Monde/Quand nasquit celle en qui mourant je vis » (D7), ou « Tant je l'aymay, qu'en elle encor je vis » (D 49), ou «En toy je vis, ou que tu sois absente:/En moy je meurs, ou que soye present » (D 144), ou encore, à l'adresse des sourcils de Délie mués en arcs, «Car qui par vous conclut resolument/Vivre en aultruy, en soy mourir commence » (D 270), le lecteur ne peut pas ne pas se souvenir de ce point de la doctrine ficinienne, dont le passage suivant donne une idée: « L'esprit doncques de l'Amoureux n'est en soy, car il n'œuvre en soymesmes. $S^{\prime}$ il n'est en soymesme, il ne vit aussi en soymesme. Et qui ne vit, il est mort. A ceste cause, quiconque ayme, est mort en soy. Ne vit il a tout le moins en aultruy? Si faict vrayement. Deux especes y a d'Amour. Un Amour est simple: 1'autre est mutuel. Amour simple, est quant cil qui est aymé, n'ayme celluy qui l'ayme. La totalement est mort l'Amoureux [...] Mais ou cil qui est aymé correspond en Amour, en ce seulement vit l'Amoureux. De ce merveilleuse chose advient, que toutes \& quantes fois que deux, quels qu'ils soyent, s'entretiennent par mutuelle beneuolence, l'ung en cestuy, et cestuy en l'autre vit. » (Le Commentaire de Marsille Ficin, Florentin: sur le banquet d'Amour de Platon: faict Francois par Simon Siluius, dit I. De La Haye..., Poitiers, 1546 [BNF Rés. p. R. 393/B.M. Toulouse Rés. D XVI. 283], f XVIII $\left.\mathrm{r}^{\circ}-\mathrm{XVIII} \mathrm{v}^{\circ}\right)$.

18. Comme le remarque G. Defaux, Marot, sur le même thème du partage des qualités de l'Aimée, trousse dans l'Adolescence clementine un rondeau (XLV) de configuration « beaucoup moins néoplatonicienne que celle de notre Amant ». Voir son édition de la Délie, Paris, Droz, 2004, t. II, p. 173. 
L'héritage néo-platonicien est donc flagrant. Pourtant, si l'intertexte est bien là, et en abondance, suffit-il à autoriser, à l'échelle du recueil dans son ensemble, la conversion de l'innutrition en orientation (c'est l'un des sens, et non des moindres, du mot « sens " précédemment mis en exergue) ? C'est loin d'être évident, et pour plusieurs raisons.

La première est qu'il existe d'autres intertextes concurrents, même s'ils sont quantitativement moins importants. Scève n'a pas tout à fait rompu, par exemple, avec la physique aristotélicienne et une psycho-physiologie galénique qui amortit considérablement la dichotomie âme/corps ${ }^{19}$; il se souvient plus souvent qu'il n'y paraît de l'acuité mordante d'un Catulle, des virtuosités des Grands Rhétoriqueurs et de la tradition épigrammatique alexandrine; et il chante souvent Délie en des accents qui lui confèrent une dimension mariale récemment réévaluée ${ }^{20}$, et difficilement compatible, malgré de superficielles affinités, avec la métaphysique platonicienne ${ }^{21}$.

La deuxième est qu'en bonne logique, l'épuration progressive devrait aboutir, fût-ce au prix de « rechutes » passionnelles difficilement surmontées, à un dépassement de la fureur amoureuse au bénéfice de la contemplation d'un Bien et d'un Beau indissociables. Or, en dépit d'une ouverture très engageante de l'ultime dizain sur la « Flamme si saincte », ce dépassement n' est pas acquis, ou du moins prend un tour ambigu: ce qu'affirme le D 449, ultime pièce, ce n'est pas une victoire d'Antéros sur Éros, de l'Idéal sur le sensible, mais leur équation, leur intime et éternelle intrication sous le commun vocable d'Amour:

Aussi je voy bien peu de difference

Entre l'ardeur, qui noz cœurs poursuyvra,

Et la vertu, qui vive nous suyvra

Oultre le Ciel amplement long, \& large.

Et le mystérieux « Genevre » final, censé vivre « Non offensé d'aulcun mortel Letharge ", fait beaucoup plus sûrement jouer le caractère sempervirens d'une plante prise pour métaphore de l'éternelle union de deux cœurs, dans la lignée du chèvrefeuille de Marie de France, qu'une vertu purificatrice ou protectrice - en tout cas, sanctificatrice -, que lui prêtent - de manière très marginale - les occurrences scripturaires ${ }^{22}$.

19. Voir les rappels de J. Céard dans « Sens, cœur, raison, mémoire dans Délie: la "psychologie" de Scève », p. 16, dans Lire Maurice Scève, Cahiers Textuel 34/44 Paris, Université Paris VII, 1987, p. 15-25.

20. Voir les nouvelles pièces versées au dossier par G. Defaux, éd. cit., t. I, p. CII-CLXIII.

21. Notamment à cause de la problématique du péché: le salut à Marie l'inscrit en figure inversée de la tentatrice immémoriale ( $E V A / A V E)$, dont le corps même est chanté comme imputrescible et signe de pureté; elle rédime et aide tous les pécheurs à n'adorer que le Créateur, qui est aussi Sauveur. Pas de péché au contraire chez Platon, rien que des erreurs, des illusions, des ombres sur les parois, et une démarche d'éveil des plus aristocratiques: au pays où Socrate lui-même avoue avoir du mal à suivre les raisonnements de Diotime, beati diuiti spiritu...

22. Sans nier l'intérêt d'une publication qui a fait date, qu'il soit ici permis de formuler de sérieuses réserves sur la pertinence même de l'intertextualité biblique dans les derniers dizains: lorsque L. K. Donaldson-Evans voit dans ce « Genevre » un écho du genévrier sous lequel va s'asseoir le prophète Élie que sa condamnation à mort par Jézabel vient de mettre en fuite (I Rois, 4-5), il faut déjà solliciter le texte pour voir dans ce végétal un symbole d'immortalité: en effet, Élie demande 
La troisième est une question de tonalité: il a été trop peu remarqué que le néo-platonisme ne fait pas bon ménage avec l'humour, et rien n'est plus désespérément sérieux que le Commentaire de Ficin sur le Banquet de Platon ${ }^{23}$. Or, comme l'ont montré plusieurs critiques depuis Saulnier, le texte de la Délie révèle assez fréquemment un Scève "gabeur », malicieux, ludique ${ }^{24}$, amateur d'une auto-dérision qui accuse la frontière entre le poète et l' Amant, facette longtemps occultée, sciemment ou non, par les partisans de l'élévation spirituelle. Il n'est pas jusqu'aux nombreux effets de distorsion entre la leçon apparente de certains emblèmes et la chute, ou l'esprit, des dizains d'accompagnement, qui ne plaide en faveur d'un jeu du poète par-dessus les affres et rémissions de l'Amant, comme si le recueil mettait en scène une parole incertaine sur le sens des signes, et donc le parcours entier à distance.

Bien sûr, une lecture néo-platonicienne concèderait la présence de « rechutes » dans l'obsession charnelle, mais à la seule condition de trouver quand même son compte dans le finale du recueil - sinon, que resterait-il de l'épuration spirituelle? Or, non seulement le D 449 conserve de façon gênante cette « ardeur » qui aurait pu ou dû disparaître ${ }^{25}$ - pourquoi ne resterait-il pas que

à mourir et s'endort sous cet arbre, puis un ange vient le toucher et lui dire de manger pour reprendre la route (« [4] et perrexit in desertum via unius diei/cumque venisset et sederet subter unam iuniperum/petivit animae suae ut moreretur et ait/sufficit mihi Domine tolle animam meam/neque enim melior sum quam patres mei/[5] proiecitque se et obdormivit in umbra iuniperi/et ecce angelus tetigit eum et dixit illi surge comede »); mais lorsque le critique ajoute que ce même végétal est évoqué au psaume 120 (Ps. 119/120,4), alors qu'il n'y est question que des flèches des ennemis de la paix, aiguisées au feu (" sagittae potentis acutae cum carbonibus desolatoriis », dit la Vulgate que pouvait lire Scève: où donc est le genévrier?), il récrit l'Ancien Testament; et lorsqu'enfin le même arbuste est censé être celui sous lequel Marie dissimule Jésus lors de la fuite en Égypte, il se met à inventer le Nouveau... car ni Matthieu, qui seul des canoniques narre l'épisode - expédié d'ailleurs en trois versets $(2,13-15)$ -, ni même les apocryphes (Pseudo-Matthieu en tête) ne parlent d'un genévrier à cette occasion. Évidemment, une fois ces menues retouches effectuées, il est toujours possible d'en conclure: « the juniper bush [...] expresses the immortality of the Vita Nova that Scève has attained in the course of his poetic and spiritual pilgrimage» ("Love Divine, All Loves Excelling: Biblical Intertextualities in Scève's Délie ", p. 14, dans French Forum, vol. 14, n¹, 1989, p. 5-15). Comble d'infortune pour cette aimable légende du genévrier, les Septante ont fait erreur en traduisant dans ces deux passages par «iuniperus » un terme hébreu qui signifie en fait " genêt », plante effectivement connue pour l'excellence du charbon qu'elle fournit, ce qui explique le rougissement des flèches comme symbole des langues perfides. Certes, Scève pouvait faire confiance à la Vulgate (et donc broder sur une erreur), mais il ne pouvait trouver ailleurs que dans ce mince passage un lien (d'ailleurs plus que douteux!) avec l'immortalité. Voir les Écrits apocryphes chrétiens, Paris, Gallimard, 1997, p. 117-140, et le Dictionnaire de la Bible, F. Vigouroux éd., Paris, Letouzey et Ané, 1903, t. III, p. 183-187.

23. Une exception notable est la question presque attristée de P. R. Blum devant le fait que Ficin gomme la dimension de disputatio des dialogues platoniciens et l'ironie comme méthode: «Ficin n'avait-il donc aucun humour? aurait-il emprunté à Platon la forme du dialogue sans en saisir du tout la méthode? » (cité par P. Laurens dans son édition du Commentaire, Paris, Les Belles Lettres, 2002, p. XLIII).

24. Voir par exemple l'atmosphère franchement comique du D 120, le jeu sur le "cerf en campaigne » au D 225, ou l'énigmatique juxtaposition de « coulpe » et « bonne adventure » au D 287. Il faudrait aussi penser aux équivoques grivoises, d'esprit assez marotique, sur le « maint cas » au D 83, ou le «Bracquemart de Mars » au D 110.

25. Cette gêne se sent chez L. K. Donaldson-Evans lorsqu'il écrit: «thanks to the purification love has undergone in his work, Scève can boldly equate « ardeur » and « vertu » » (art. cit., p. 13). La purification aurait été plus concluante s'il n'y avait plus eu aucune équation à faire... 
la Vertu pour et dans l'éternité? -, mais les deux dizains qui le précèdent, et qui doivent être considérés comme faisant un tout avec lui, puisque Scève isole ostensiblement ces trois-là, et parce qu'ils ne sont que trois, à la suite du dernier emblème, sont loin d'assurer le triomphe de l'esprit sur la chair et de la contemplation béate des essences sur la rage libidinale. Le D 447 se termine sur ce constat de l'Amant:

Qu'apres ma mort encores cy dedens

Je pleure, \& ars pour ton ingratitude.

Et le D 448, dernier dizain "politique », curieusement situé en un endroit où le lecteur attendrait un détachement total des affaires mondaines, paraît bien viser l'outrecuidance d'un Amant qui aurait présumé de ses forces:
Vouloir tousjours, ou le povoir est moindre,
Que la fortune, \& tousjours persister
Sans au debvoir de la raison se joindre,
Contre lequel on ne peult resister,
Seroit ce pas au danger assister,
Et fabriquer sa declination?
Seroit ce pas, sans expectation
D'aulcun acquest, mettre honneur a mercy,
Ou bien jouer sa reputation
Pour beaucoup moins, qu'a Charles Landrecy?

La référence à l'humiliante déroute de Charles Quint devant Landrecies en 1542 suggère, par un effet d'hyperbole analogique, que l'Amant a tout simplement risqué sa ruine sociale - voire celle de l'Aimée ${ }^{26}$ - en s'obstinant dans sa quête, que le but de celle-ci soit la conquête entière de Délie ou au contraire le renoncement définitif à celle-ci. La « flamme si saincte » du tout dernier dizain sonne ipso facto, sinon comme une solution par défaut, du moins comme un rattrapage in extremis, voire une victoire à la Pyrrhus.

Mais il y a mieux, ou pire: si le sens détermine l'ordre, si donc une élévation spirituelle se dessine progressivement, du plus charnel au plus idéal, que dire des dizains qui eussent fait une rayonnante conclusion, bien dans l'esprit néoplatonicien - ou même simplement chrétien, car à ce stade les deux orientations font cause commune et partagent les mêmes intérêts ${ }^{27}$ - et se situent néanmoins dans le cours du recueil, comme si un malin génie les avait interpolés? Deux cas sont particulièrement frappants: au plus près, le D 446 s'achève sur une magnifique ouverture à une existence meilleure et de plus haut sens, d'autant

26. C'est l'opinion de G. Defaux, éd. cit., t. II, p. 485. L'absence de rupture dans les anaphoriques et le régime percontatif des deux questions successives laisse néanmoins entière la possibilité d'un discours exclusivement consacré aux enjeux du côté de « je »: $1^{\prime}$ « honneur » et la « reputation » dont il est question peuvent très bien se rapporter à l'image sociale de l'Amant. Mais les deux options sont indifférentes quant aux conséquences sur la dimension spirituelle.

27. Comme le rappelle P. Laurens (éd. cit., p. XLI) : « Célébrant l'amour divin, Ficin obéit [...] à sa mission providentielle qui est de ramener à un christianisme ardent par la raison platonique les esprits réticents au message de la révélation ». C'est P. Laurens qui souligne. 
plus en contraste avec le D 448 qu'elle adopte la même tournure de question oratoire:

Doncques, pour paix a ma guerre acquerir,

Craindray renaistre a vie plus commode?

Quand sur la nuict le jour vient a mourir,

Le soir d'icy est Aulbe a l'Antipode.

Quelle plus belle auto-exhortation au courage (d'en finir avec le désir érotique) que cette reprise quasi littérale de l'analogie tissée par Speroni dans le premier de ses Dialoghi d'Amore ${ }^{28}$ ? Et quel apaisement que cette clôture gnomique au rythme fluide, qui contraste avec la persistance du questionnement et les rêches enjambements du D 448! Comme l'a fort bien remarqué L. K. DonaldsonEvans, c'est sur ce dizain que le «cycle » de l'aventure passionnelle se termine effectivement ${ }^{29}$. Ou plutôt devrait se terminer, car les trois dizains qui suivent sont décidément bien encombrants, et qu'il ne servirait à rien de voir dans l'emblème final, «le Tumbeau \& les Chandeliers », une séparation critique situant désormais la parole de l'Amant outre-tombe. Le remède serait même pire que le mal: une fois la mort acquise, sur quelque plan qu'il faille la comprendre, pourquoi se soucier encore d'ingratitude, de poliorcétique et d'ardeur?

Au plus loin maintenant, à un huitième du recueil, le $\mathrm{D} 56$, qui sonne à la fois comme une récapitulation et comme une (heureuse) capitulation:

Le Corps travaille a forces enervées,

Se resolvant l'Esprit en autre vie.

Le Sens troublé voit choses controvées

Par la memoire en phantasmes ravie.

Et la Raison estant d'eulx asservie

(Non aultrement de son propre delivre)

Me detenant, sans mourir, \& sans vivre,

En toy des quatre à mis leur guerison.

Doncques a tort ne $t^{\prime}$ ont voulu poursuyvre

Le Corps, l’Esprit, le Sens, \& la Raison.

Le lecteur y retrouve sans peine l'idée ficinienne de la vie en autrui, dans cet être aimé qui décide de tout, ainsi qu'une sage hiérarchie progressive qui mène du corps à la raison, en passant par les esprits (globalement, les facultés vitales sensitives) et le sens, qui ne peut plus juger sainement de ce qui lui revient en mémoire à cause de l'affaiblissement physiologique ${ }^{30}$. Le passé composé final complète à merveille, par son aspect accompli, le présent qui jusque-là généralisait l'expérience. Le poète va même jusqu'à n'inscrire la personne de l'Amant que de façon fugace (au v. 7), avant de conclure dans l'absolu, ou l'universel,

28. « La carne \& l'ossa, di che noi siamo formati, con la loro imperfettione sono cagione di farci sentire di quegli effetti miracolosi: che com'hora non è giorno per tutt'il mondo, ma il nostro uespro è mezza notte ad altrui, \& la sera di questo hemisperio è l'alba dell'altro » (cité par Mc Farlane dans son éd. de la Délie, Cambridge, C.U.P., 1966, p. 485).

29. Art. cit., p. 12.

30. Voir J. Céard, art. cit., p. 16-18. 
des quatre facultés en jeu. Le bilan est ici très clair: dans un renversement prédicatif qui rend justice à la qualification d' « epygrammes », la somme des avanies apparentes doit être comprise, au terme de tant d'efforts, ad maiorem deae gloriam, et l'Amant se réjouit de trouver le remède au sein même du mal. Cette auto-approbation sans remords ni regrets n'a peut-être pas la profondeur du D 449 , due en partie à la promesse d'un verbe au futur et à sa malicieuse intertextualité ${ }^{31}$, mais elle pourrait sans conteste prendre place parmi les tout derniers dizains - en tout cas bien après l'évocation très « terre-à-terre » de la bataille de Landrecies. Non seulement personne n'y aurait trouvé à redire, mais l'interversion de ces deux dizains aurait fourni des armes considérables aux tenants de la lecture spiritualisante ${ }^{32}$.

La seconde hypothèse - celle d'un parcours non pas linéaire mais cyclique - peut être examinée plus rapidement, à la fois parce que la littérature critique est plus mince et plus hésitante à son sujet, et parce que la notion de circularité, passé le charme de sa perfection géométrique, se heurte à un problème de définition: dès lors qu'un retour pur et simple du discours poétique à l'initus a quo du recueil (huitain liminaire ou $\mathrm{D} 1$, là encore il faut trancher) paraît exclu - s'il y a promesse d'immortalité au D 449, il y a gain au terme d'un parcours, et dans cette perspective la "Flamme si saincte " peut faire figure d'Aufhebung par rapport à la Dame «Idole de ma vie » comme aux «mortz » renouvelées, - il est fort difficile de fournir des critères sûrs de vérification. Sur quel plan,en effet, situer le trajet du recueil? Sitôt quitté le domaine du lexique ou de la thématique manifeste, d'autant moins suffisant que l'enjeu d'un texte peut provenir précisément d'une impasse, d'un non-dit, de l'absence concertée d'un

31. Avec le Pétrarque du Trionfo del Tempo, ce qui referme doublement la boucle ouverte par le D1. Voir Parturier, éd. cit., p. 306, et infra.

32. L'interversion est d'ailleurs miraculeusement satisfaisante dans les deux sens: car si le D 56 peut conclure le recueil pour les raisons susdites, le remplacement de sa teneur par celle du D 448 entre le D 55 et le D 57 aurait ravi les amateurs de cohérence et de continuité, en renforçant une série «politique » (le D 53 vante la libération de François Ier, le D 54 et le D 55 flétrissent les ambitions de Charles Quint - « L'hoir de Jason », puis « L'Aigle » transmué en « Austruche errante », et « Charles » enfin nommé aurait bouclé la boucle en faisant écho au « FRANCOYS » du D 53, tout en conservant, ce qui n'est pas rien, la chronologie des faits évoqués). Last but not least, la fin du D 448 fournirait au D 57 une transition lexicale et thématique que n'assure absolument pas le D 56, avec le motif du jeu (de « jouer sa reputation », v. 9, à « celluy, qui jouant a la Mousche », v. 1), et une parenté certaine dans la «leçon " (l'auto-aveuglement). Bref, Scève aurait délibérément mis un dizain à la place de l'autre qu'il ne s'y serait pas pris autrement... Un lecteur taquin pourrait même s'amuser à généraliser les tentatives d'interversion. Dans certains cas, celle-ci est possible entre deux dizains immédiatement successifs: ainsi des D 395 et 396, qui évoquent le confluent lyonnais. Non seulement l'anaphorique du dernier vers du premier ("Ce mariage entre eulx») serait plus clair si le Rhône avait déjà été nommé (il l'est au v. 5 du second, comme l'est "Arar », la Saône, mais toute seule, au v. 4 du premier, où de surcroît deux autres anaphoriques, «celle » et "ceste", compliquent la référence), mais l'ouverture du D 396, qui à la figure du laboureur revenant des champs fait succéder celle du «Pelerin, son voyage accomply », qui « Retourne en paix », reprendrait par antithèse le motif final du D 394, où l'Amant voit Délie «a [s] es vœutz se rendre inexorable». 
terme ${ }^{33}$, il faut bien aborder celui de l'interprétation, qui suppose l'orthodoxie d'une lecture tenant compte et de l'intertextualité interne et de l'intertextualité externe. Mais même lorsque celle-ci fait l'unanimité - il est patent par exemple que le D 449 revient à Pétrarque, comme le D 1 commence avec lui ${ }^{34}$-, il reste à apprécier à la fois la distance prise avec le ou les textes sollicités, et, surtout, la portée, la zone d'influence du dizain considéré.

$C^{\prime}$ est ainsi, par exemple, et pour rester sur ces emplacements stratégiques de l'ouverture et de la clôture du recueil, que l'hommage de la reprise quasi textuelle s'accompagne, dans les deux cas, d'une véritable réécriture du prédicat pétrarquien ${ }^{35}$. Il y a donc distance à chaque fois, encore plus sensible pour les happy few de l'époque. Mais qu'en est-il de la distance entre ces deux distances? La sacralisation de l'Amour telle qu'elle apparaît in fine, avec sa réunion de l'Amant et de l'Aimée dans un "nous » irénique - et à supposer qu'il recouvre bien le «je » de l'Amant et le « elle » de l'Aimée, ce qui n'est pas démontré -, est-elle vraiment jouée dès le récit de l'innamoramento, qui place face à l'« Idole » une "piteuse hostie " plus qu'à son tour harassée de son « si long service » (D 438), et donc instaure une position d'infériorité? En est-elle la suite prévisible, l'aboutissement de fait, ou bien le but envisagé comme un vœu pieux? Si de nombreux critiques, depuis Saulnier, ont vu dans l'ultime pièce de la Délie une assomption réussie et comme un havre de paix qui ne retournait sur les traces pétrarquiennes que pour faire mesurer le chemin parcouru, il est également permis d'y voir un moment d'optimisme qui en rappelle d'autres, et reste sujet à caution ${ }^{36}$. En d'autres termes, une boucle peut fort bien se refermer sur

33. Ce ne sont pas là rêveries «modernes », oulipiennes ou borgesiennes, trop souvent traitées avec condescendance: Ficin n'emploie pas une seule fois le mot «baiser » dans tout son Commentaire... Quand le lipograme n'est pas un jeu - et Ficin ne joue pas -, il est l'expression d'un tabou.

34. La formule «en mes jeunes erreurs» du D 1 reprend le «in sul mio primo giovenile errore » du premier sonnet du Canzoniere (I, 3), et le « mortel Letharge » qui clôt le D 449 reprend le « grave e mortifero letargo » du Trionfo del Tempo (v. 75).

35. L'adoption du pluriel à la place du singulier se justifie notamment par la torsion de la «leçon » du poème inaugural: chez Pétrarque, l'«erreur juvénile» n'est autre que la passion pour Laure, désormais dépassée par la conscience du caractère éphémère des choses de ce monde, alors que Scève laisse entendre une pluralité de liaisons ou d'attachements séchement interrompue par la révélation d'une « Dame » qui devient « Idole ». La metanoïa joue donc quasiment à rebours. Quant au « Letharge » final, il suffit de se rappeler que, chez le devancier toscan, c' est l'oubli mortel effectivement promis à l'amour qui doit s'incliner devant le Temps, alors que Scève inverse le triomphe, puisque le "Genevre", précisément, ne sera pas offensé. Voir respectivement JoAnn Della Neva, Song and Counter-Song: Scève's "Délie » and Petrarch's "Rime», Lexington, French Forum Monographs, 1983, p. 85 sq., et T. Cave, "Scève's Délie: Correcting Petrarch's Errors », Pre-Pleiade Poetry, J. C. Nash éd., French Forum, 1985, p. 112-124.

36. G. Defaux va plus loin, en remarquant que « cette projection triomphante vers le futur vient tout juste après le moment de doute et d'interrogation que constitue D 448. Rien donc n'est plus fragile que cet envol vers l'Éternité. Il n'a que l'épaisseur d'un moment, du temps qu'il a fallu à l'Amant pour le concevoir, et au Poète pour en faire un dizain. Nous savons, nous qui avons lu les quatre cent quarante-huit dizains qui précèdent, que ce moment sera suivi par d'autres [...] que la colère, la jalousie, l'amertume, le découragement, feront leur réapparition. Il s'agit donc de ne pas être dupe, de se dire que ce n'est pas vraiment la fin » (éd. cit., t. II, p. 486). 
un certain plan - l'allégeance ironique ou subversive à Pétrarque - et pas sur un autre, celui de la tonalité et de la leçon initiales.

Il semblerait d'ailleurs que ce composé de symétrie et d'asymétrie, de répétition et de variation, de circularité et de décalage, soit à l'œuvre dans une autre catégorie $d$ 'indices structurellement signifiants, auxquels il est impératif de se reporter dès qu'il est question de circularité: les emblèmes. Leur caractère hétéroclite, tant en notoriété du motif qu'en noblesse de l'univers de référence - « du phénix au pot-au-feu », pour reprendre le titre d'une célèbre contribution $^{37}$ - présente apparemment un rassurant correctif dans la régularité de la succession des cadres géométriques encerclant les vignettes: au rectangle succède un cercle, puis un losange, puis un ovale horizontal, puis un triangle équilatéral, et enfin un ovale vertical, avant que ne revienne le rectangle. C'est un dispositif étrange, mais qui ressemble bien à un ordre significatif, et qui autorise à première vue l'hypothèse d'un roulement entre six «familles » de neuvaines, qui auraient chacune leur couleur, leur sonorité privilégiée, voire leur thématique. Il faut pourtant en rabattre, même si l'option est prise de considérer que Scève a tout contrôlé de l'insertion des emblèmes, et cela pour trois raisons.

Tout d'abord, il n'y a pas vraiment six variétés de cadre, mais « cinq plus une ». En effet, l'ovale vertical est le seul qui conserve strictement la même décoration, alors que celle-ci se renouvelle systématiquement pour les cinq autres cadres. Impossible, jusqu'à présent, de justifier ce caractère exceptif, de trouver le sens de cet ordre.

Ensuite, la régularité de rotation des cadres n'est pas absolument parfaite: l'emblème 31, qui devrait logiquement être encadré par un rectangle, l'est par un ovale horizontal, et le rectangle reparaît immédiatement après, ce qui signifie au moins que la série est close sur cette substitution - sinon, le lecteur trouverait un triangle, puis un ovale vertical, avant de retrouver le rectangle. Ce décalage est-il dû au souci de ménager la place finale au rectangle, pour produire un écho dès lors cyclique au tout premier emblème, qui était aussi un rectangle? Mais, si tel est le cas, d'autres solutions existaient qu'une infraction à cette impeccable rotation: une modification du nombre de types de cadre si Scève tenait à garder le même nombre total de dizains, ou à l'inverse un changement du nombre des dizains s'il tenait à celui des variétés de cadres.

Enfin, un problème du même ordre est posé par l'ultime série, où le rigoureux agencement est à nouveau perturbé - ce qui jette le trouble sur les motivations du décalage précédent. La succession y devient en effet: rectangle - cercle - losange - ovale vertical (et non horizontal) - triangle - cercle (une seconde fois, au lieu de l'ovale vertical) - rectangle ${ }^{38}$. Seule « cohérence » à l'intérieur de cette incohérence, il est patent que la figure manquante ici correspond à

37. F. Lecercle, art. cit.

38. Voir les remarques de F. Joukovsky dans son édition de la Délie, Paris, Dunod, «Classiques Garnier », p. XLIV-XLVII. 
la figure surnuméraire là-bas, et qu'ayant déjà été répété "indûment » entre l'ovale vertical et le rectangle à l'emblème 31 , l'ovale horizontal n'est plus « disponible " pour la dernière série. Mais pourquoi ce choix, qui a au minimum pour conséquence de compromettre la structure cyclique dans son extension à la série d'emblèmes, étant donné que la première série ne trouve alors plus son écho à la fin du recueil ? Serait-ce pour limiter la circularité au rectangle, donc au niveau de la symbolique d'un seul des emblèmes? Mais alors pourquoi ne pas avoir choisi précisément le cercle dans cette fonction, qui redoublerait, comme par une mise en abyme, la notion de circularité ? $^{39}$ Et comment expliquer les autres modifications dans cette ultime série, qui paraissent attirer l'attention sur un niveau supérieur en produisant un chiasme géométrique presque parfait $?^{40}$

Par ailleurs, au-delà de ces trois obstacles matériels, l'hypothèse d'un parcours cyclique, dès lors qu'elle doit tenir compte des emblèmes (jusque dans leur ornementation), achoppe sur un problème sans doute encore plus redoutable, qui est celui du lien entre ces emblèmes et le texte, ne serait-ce qu'au seul niveau du rapport entre le cadre géométrique et le couple sémiologique imprese-motto. De même que la démonstration a été faite de l'impossibilité d'assigner aux emblèmes une fonction discursive univoque, stable, par rapport au texte des dizains, et que le mélange de reprises et de ruptures, parfois même implicitement ironiques, entretient à l'évidence une « mosaïque » déstabilisatrice ${ }^{41}$, de même il est illusoire de vouloir assigner à tel type de cadre tel type d'image, de genre ou de leçon.

Pour ne prendre que deux exemples, le fameux rectangle initial et final paraît dans ses quatre premières apparitions dévolu à des figures mythiques assez prestigieuses, ou du moins solliciter un intertexte (païen ou chrétien) de haute volée, et qui plus est en relation directe avec le couple Éros/Thanatos: la licorne, Narcisse, Didon, Actéon. Las! la quatrième vignette est l'une des plus triviales et farcesques du recueil, celle de "La Selle, \& les deux Hommes », où un farceur retire une chaise au moment où quelqu'un veut $s^{\prime} y$ asseoir. Et, inversement, l'ovale horizontal paraît au départ se spécialiser dans l'évocation des travaux et des jours, dans l'humilité de realia quotidiens, avec une certaine redondance: «L'homme \& le Bœuf », « Deux Bœufx a la Charue », «La Cycorée », « Le Bateau a rames froissées », « Le Forbisseur ». Mais ensuite se produit

39. En choisissant le rectangle pour encadrer le recueil alors même qu'il montre qu'il dispose du cercle, Scève désolidarise la circularité de la perfection et de la paix: des deux figures, c'est bien le cercle qui montrerait le mieux un achèvement réussi; le rectangle, lui, connote davantage le conflit.

40. Aux extrêmes, deux rectangles; un cran à l'intérieur, en deuxième et avant-dernière position, tout aussi symétriques donc, deux cercles; et, un second cran à l'intérieur, de part et d'autre de l'ovale vertical central, un losange et un triangle. Ces deux dernières figures diffèrent, certes, mais elles épuisent, en correspondance avec le rectangle, les figures angulaires. Le chiasme recouvre donc en fait aussi une alternance entre brisure et continuité linéaires.

41. Voir les pénétrantes analyses de C. Alduy, « Délie en mosaïque », Poétique, n 127, sept. 2001, Paris, Seuil, p. 281-300. 
la perturbation de l'emblème 31, et le cadre orne alors « Le Papillon \& la Chandelle », ce qui est déjà un peu moins prosaïque; l'ordre paraît revenu à l'occurrence suivante, puisque l'ovale horizontal encadre alors "L'Asne au Molin », mais tout s'écroule à la suivante, qui propose « Leda \& le Cygne ». Il n'y a donc manifestement pas plus de cohérence thématique ou prédicative à l'intérieur d'une variété donnée de cadres qu'il n'y en a à l'intérieur des « neuvaines » de dizains ${ }^{42}$.

Dernier élément susceptible de fragiliser l'idée de circularité: si, comme l'a montré le développement précédent, l'hypothèse d'une progression spiritualisante stricto sensu - linéaire et pleinement satisfaisante in fine - est plus que discutable, il est néanmoins possible d'observer certains itinéraires sectoriels, certaines modifications du discours du poète au fil du recueil, tantôt sur un thème précis, tantôt sur une tonalité ou un principe de construction de l'épigramme. Très concrètement, cela veut dire, par exemple, que les métaphores de la maladie et de la guerre, fort proches dans leurs connotations et leurs contextes d'emploi, car toutes deux renvoient au désordre intérieur de l'Amant, subissent une inflexion rien moins qu'innocente, sans doute, au fil du recueil, dans la mesure où les vocables qui désignent l'une et l'autre font progressivement l'objet d'une « appropriation » par le locuteur, la «fiebvre » devenant plus fréquemment «ma fiebvre », et «la guerre » « ma guerre » une fois passée la moitié du recuei ${ }^{43}$. Cela veut dire aussi que, si sont examinés à part, comme une catégorie de dizains particuliers, les dizains dialogués, où la parole est donnée à un personnage, fictif ou non, puis au moins à un autre qui réplique, une évolution se dessine quant au maniement de la parole vive, à savoir le gain croissant d'une supériorité de l'Amant, sur le terrain de la vérité, par rapport aux deux autres principales instances que sont l'Aimée et l'Amour personnifié, supériorité qui apparaît comme une compensation épigrammatique de son infériorité sur le plan existentiel ${ }^{44}$.

Bref, le recueil présente juste assez de marques évolutives pour qu'il soit exclu de lui trouver une structure cyclique digne de ce nom, et un peu trop de marques de circularité, de reculs ou de piétinements pour que s'impose sans accroc l'idée d'une progression linéaire. Mais, dès lors, si aucun de ces trajets n'est satisfaisant, c'est peut-être qu'ils pèchent en tant que trajets, et que le recueil n'obéit à aucun autre principe de structuration que celle d'un réseau fait

42. Et l'emblème ne «lance» pas la tonalité de la neuvaine à partir du dizain d'accompagnement: E 29, « La Cye », avec sa devise " Force peu a peu me mine », ouvre le thème du découragement; mais dans cette même série prend place le D 264, qui affirme de façon triomphante que les dédains de Délie sont sans effet sur la constance de l'Amant. Même contraste entre l'E 32, « Le Muletier » (devise: « Double peine a qui pour aultruy se lasse »), et le D 287, troisième de la neuvaine concernée, allusion réjouie à une «bonne adventure ». Les exemples abondent.

43. Un travail de doctorat en cours, sur la métaphore dans la Délie, précise et exploite ces constatations.

44. Voir notre étude « Les dizains dialogués dans la Délie de Maurice Scève », Bibliothèque d'Humanisme et Renaissance, LXIV, n 3, Genève, Droz, 2002, p. 579-604. 
de nombres et/ou de proportions. C'est là la seconde grande option qu'il était possible d'envisager, et qu'il est temps de mettre à l'épreuve.

Une première possibilité se dégage d'un constat de prime abord fort prometteur: à mi-recueil, le D 224 exprime à satiété une nouvelle naissance de l'amour, dans une sorte de printemps tardif qui réveille les ardeurs assoupies d'une première passion:

Novelle amour, novelle affection,

Novelles fleurs parmy l'herbe novelle:

Et, jà passée, encor se renovelle

Ma Primevere en sa verte action.

Plus sombre, la suite du dizain met en vedette «la renovation » de l' « ulcere ancienne " de l'Amant, comparable en douleur à une "playe Egyptienne » à lui infligée par un «meurdrier » qui ne peut être que le dieu Amour. Le martèlement de la racine [nov-] dans tout le poème, de l'adjectif trois fois répété au substantif en passant par le verbe pronominal, semble indiquer avec force un effet de structure à l'échelle de l'ensemble du recueil; et si, sur cette lancée, le découpage en deux moitiés incite le lecteur à rechercher une subdivision confirmatrice, celui-ci peut se réjouir de voir qu'à mi-chemin de ce premier volet du diptyque, le D 112 évoque déjà cette même thématique d'un changement d'époque, et en des termes si voisins que le hasard doit être exclu: l'Amant végétait autrefois dans une bien curieuse ataraxie, "Tout deschargé des amoureuses sommes » (v. 4), jusqu'à ce que «la Fortune envieuse/Trouble [s] a paix par troys lustres joyeuse » (vv. 6-7). Même le lexique du D 224 est déjà présent, avec un « Renovellant » (v. 8) et une âme « toute playeuse » (v. 9) qui ne trompent pas. $\mathrm{Si}$, comme le note $\mathrm{G}$. Defaux, une subtile progession distingue les deux dizains, ils restent pourtant liés de manière évidente en suggérant « une structuration voulue $»^{45}$.

Et inévitablement, par la force même d'un esprit de symétrie qui trouve ici sa pâture, le lecteur s'empresse de vérifier, de l'autre côté du diptyque, la présence d'un pendant du D 112, qui ne peut être arithmétiquement que le D 336. Hélas, le fil rouge lexico-thématique du renouvellement de la blessure amoureuse n'est pas au rendez-vous, car ce dizain déplore simplement les suites funestes d'un attachement à l'« Enfant desvoyé » qui fait redouter à l'Amant une égale détention de son corps et de son cœur. Et ce qui dissuade de forcer le texte de ce dizain pour y trouver coûte que coûte des échos des deux précédents, c'est qu'à trois unités de distance, avec une légère anticipation, le D 333, lui, assure ce lien de manière flagrante: 1 'Amant jette un regard sur de longues périodes de son existence, et regrette que sa « courbe viellesse accule/Celle verdeur » qu'il avait sentie «novelle». Et d'ajouter, comme si la correspondance lexicale devait être soulignée:

45. Éd. cit., t. II, p. 260. 
Ce neantmoins tousjours se renovelle

Le mal, qui vient ma playe reunir.

Si donc l'équilibre structurel est globalement respecté, l'incontestable décalage arithmétique est frustrant ${ }^{46}$, d'autant qu'un regard d'ensemble sur les dizains 330 à 338 - la neuvaine de l'emblème XXXVII, « La Lune en tenebres » - fait apparaître l'absence de tout obstacle à l'interversion des D 333 et D $336^{47}$. Scève se serait-il ingénié à brouiller les pistes, en ne respectant qu'à demi un ordre embryonnaire? C'est ce que semble confirmer un autre détail troublant de la zone médiane du recueil: logiquement, le vrai milieu d'un ensemble de 449 poèmes est le deux cent vingt-cinquième, puisqu'un nombre égal de poèmes l'entoure. À ce compte, le D 224 serait en réalité conclusif tout en annonçant un nouveau départ, et le D 225 serait, lui, le véritable pivôt central, un janus bifrons partageant rigoureusement le recueil en deux masses équivalentes. Or, ce dizain débute ainsi :

Libre je vois, \& retourne libere

Tout Asseuré, comme Cerf en campaigne...

L'écho très sensible de l'incipit du D 6 ("Libre vivois en l'Avril de mon aage ») risque fort d'être en trompe-l'œil, dans la mesure où ce qui était récit d'une prise au piège amoureux de sa candeur juvénile ${ }^{48}$ devient ici fringante affirmation d'une sécurité de l'Amant que «Vertu heureuse, \& fidele compaigne » aide à lutter contre le doute et la crainte. Mais, surtout, le premier vers sonne bizarrement, avec son chiasme [adjectif - verbe - verbe - adjectif] qui semble refermer la boucle d'un processus et sa redondance lexicale légèrement archaïsante («libere » apparemment simple variante de «libre») qui ne peut qu'attirer l'attention sur l'éventuelle nuance sémantique entre les deux termes... et permettre au lecteur de se souvenir que « libre » est peut-être ici une variante francisée de «libra ${ }^{49}$, la balance latine symbole d'équilibre - avec l'appui du signe zodiacal correspondant à l'équinoxe d'automne ${ }^{50}$ - et, plus indirectement, de justice $^{51}$. Ce ne serait pas la seule fois que Scève adapterait un terme latin dans

46. Tout est affaire de degré d'exigence. G. Defaux remarque le décalage sans en déduire l'invalidité de la symétrie: «On ne peut que remarquer l'architecture, la dispositio concertée du recueil. Nous sommes avec ce dizain presque aux $3 / 4$ du parcours $(112 / 224 / 336 / 448)$. Il convient donc, pour bien lire D 333, de le rapprocher de D 112 et D224. » (ibid., p. 372).

47. Avec son thème de $1^{\prime}$ « Enfant desvoyé », le D 336 aurait succédé sans encombre au D 332, dizain dialogué sur l'anecdote du dé à coudre, et réciproquement l'évocation d'un «Amour " qui peut « les plus vieulx rejeunir » en réveillant leur mal à la fin du D 333 aurait fourni une suite plausible aux plaintes de l'Amant sur le thème d'une Dame insensible à ses pleurs. L'interversion n'aurait pas donné une séquence plus logique, mais au moins aussi logique.

48. «... soubz celle adolescence,/Ou l'œil, encor non expert de dommage,/Se veit surpris de la doulce presence » de Délie, à qui Cupidon «à toute asservie » la « liberté » de l'Amant (vv. 2-4, 8).

49. Bel et bien recensée par F. Godefroy dans son Lexique de l'Ancien français (J. Bonnard \& A. Salmon éd., Paris, Champion, 2003).

50. Voir par exemple Virgile, Géorgiques, I, 208. À noter d'ailleurs que Brunet Latin emploie le terme « libre » pour désigner ce signe.

51. La Vulgate (Proverbes, XI, 1; 20, 23) emploie le mot statera. 
le recuei ${ }^{52}$. Mais dans cette hypothèse, de prime abord très fantaisiste, quel rapport avec le reste du dizain? Ce rapport existe, et il est triple: d'abord, ce premier vers peut s'entendre comme le récit en raccourci, au présent de narration, d'une épreuve dont l'Amant ressort sans encombre (il "retourne libere ", revient du tribunal en homme libre); ensuite, il débouche sur l'ambivalente figure du "Cerf en campaigne », symbole chrétien tout autant que parangon de la luxure ${ }^{53}$, qui conforte, ou sédimente, la thématique d'une concurrence presque indécidable de polarités; enfin, et surtout, la suite et la fin du dizain filent comme par hasard la métaphore du procès, l'une des plus rares du recueil (les termes pertinents sont mis en italiques):
Selon qu'Amour avec moy delibere,
Mesmes qu'il veoit, que Vertu m'acompaigne,
Vertu heureuse, \& fidele compaigne,
Qui tellement me tient tout en saisine,
Que quand la doubte, ou la paour sa voisine,
M'accuse en rien, mon innocence jure,
Que souspeçon n'à aulcune racine
Là, ou le vray conteste a toute injure.

Il y a donc de fortes chances que Scève joue sur la double étymologie liber/libra (ou, au minimum, réveille doucement un homonyme assoupi) pour faire de ce dizain la « flèche » d'une balance qui maintient à égalité de poids les deux versants du recueil, et signifie implicitement à la fois la persistance d'un libre-arbitre et celle d'une division intime. Un élément formel supplémentaire confère d'ailleurs à ce dizain une importance particulière: face à l'aspect spectaculaire du D 224, qui disait le renouvellement de la douleur d'amour, le D 226, placé de l'autre côté de cette charnière, et au contenu prédicatif beaucoup plus ordinaire, se signale par un système de rimes uniformément masculines, qui fait contraste - et donc doublet complémentaire - avec celui du D 225, en rimes cette fois exclusivement féminines - inutile de préciser que tous les autres dizains de la neuvaine mêlent les deux types de rimes ${ }^{54}$. Autrement dit, même en dehors de

52. Comme en témoignent les «s'absconsant » (D 106), «caligineux » (D 178), « fruition » (D 226), « conqueste » (D 287), etc. En glosant ce dernier mot, G. Defaux extrapole à bon escient: « Ne pas s'étonner que Scève parle ainsi latin en français: son plus proche collaborateur à Lyon est Étienne Dolet, le cicéronien auteur des volumineux Commentaires sur la langue latine "; et d'ajouter, à propos du verbe « accusay » présent au même dizain, « accuser est l'un des sens du latin queror, conqueror. Une raison de plus de penser que l'ambiguïté voulue de ce dizain est de nature linguistique: elle tient à la tension partout présente entre le latin et le français. » (éd. cit., t. II, p. 328-329).

53. Voir les explications étoffées de G. Defaux, éd. cit., t. II, p. 262-263.

54. Qu'il ne s'agisse pas d'un hasard, mais à tout le moins d'un marqueur d'emplacement stratégique, est confirmé par une prospection de part et d'autre du D 225: en amont, le plus proche dizain à afficher pareille particularité est le D 204, où les rimes sont uniformément féminines; en aval, c'est le D 254, lui aussi en rimes uniformément féminines. La proportion des écarts est comparable, et, surtout, la distance absolue est considérable, aussi bien qualitativement (cinquante dizains d'écart entre deux dizains monorimes non « annulés » par un complémentaire, écho des cinquante emblèmes?) que quantitativement. 
toute symbolique facile, le D 225 a partie liée avec celui qui le précède comme avec celui qui le suit, mais sur des registres différents.

Néanmoins, toute tentative de convertir cette disposition objective en dispositif signifiant se heurte à de sérieux obstacles: la liberté ici claironnée fait long feu, la métaphore du cerf ne revient plus ensuite que dans une version pessimiste et doloriste ${ }^{55}$, et celle du procès réveille le motif classique de l'heautontimoroumenos ${ }^{56}$. Surtout, ce que laisse concrètement attendre cette partition en diptyque au niveau des différences discursives entre ses deux volets - une affirmation au moins plus sensible de l'innocence et/ou de la liberté de l'Amant - n'est pas vérifiable, comme si le dizain médian n'avait été qu'un feu de paille. Le lecteur se retrouve donc, par élimination d'un parcours digne de ce nom, en présence de démarcations qui ne disent pas grand-chose du territoire qu'elles bornent, ou d'un rythme sans mélodie.

Peut-être alors faut-il saisir une seconde possibilité, qui ménagerait l'existence de dizains-vedettes tout en donnant son poids à chaque élément de la séquence. En dehors de sa particularité de nombre premier, de l'intérêt de laquelle il faut bien avouer la minceur, si abondants sont ceux qui sont plus remarquables et riches de résonances symboliques à pareille échelle, 449 semble revêtir une valeur particulière depuis qu'a été remarquée l'étrange relevé de Lefèvre d'Étaples sur le nombre d'occurrences du nom de Jésus Christ dans les épîtres de saint Paul ${ }^{57}$. Débouchât-elle sur l'inexactitude de ce relevé, une scrupuleuse vérification serait de toute façon inutile: un poète peut se fier à une donnée erronée, erreur de traduction ou de comptage, pour construire son recueil. La vraie difficulté consiste à savoir dans quelle mesure cette correspondance numérique, qui n'est sûrement pas l'effet du hasard, peut être prolongée dans le détail. Il est en tout cas vite vu que l'application maximale - chacune des occurrences successives du nom de Jésus Christ, selon son contexte dans les épîtres, donnerait sa tonalité ou sa thématique au dizain correspondant - est absolument indéfendable ${ }^{58}$. Et que faire de l'application minimale - Scève ferait implicitement comprendre au lecteur que Délie est le sauveur d'un Amant qui

55. Voir les D 352 et 422 , où l'Amant se compare au cerf blessé à mort.

56. Au D 394, «J'ay mon proces contre moy intenté ».

57. « [Saint Paul] estoit si plain de Jesuchrist que tout ce qu'il pensoit estoit Jesuchrist, tout ce qu'il parloit, Jesuchrist. Quatrecens quarante neuf fois ou plus, il a en ses epistres nommé le nom de Jesuchrist » (Le Nouveau Testament, fac-simile de la $1^{\text {re }}$ éd. de Simon de Colines (1523), Paris/La Haye, Mouton, 1970, "Epistre exhortatoire ", $\mathrm{f}^{\circ} \mathrm{VI} \mathrm{r}^{\circ}$ ). Le propos est du reste remarquable par son caractère presque auto-contradictoire (précision maniaque du total suivie d'un aveu implicite de marge d'erreur).

58. Outre l'invraisemblance d'un ajustement reconnaissable de la vaste palette discursive scévienne au syntagme paulinien, aux emplois beaucoup plus « mécaniques », il reste l'obstacle du domaine de définition: que faire des « Jésus » non accompagnés de «Christ » (en I Cor., 12, 3 par exemple) ou de l'inverse (id., 15, 3 sq.) ? S'agit-il de l'expression de cette appellation précise ou de la personne qu'elle recouvre mieux ou plus fréquemment que les autres? 
pour le coup se fait aussi poète -, sinon en déduire le caractère subversif de la référence, le recueil chantant un amour indiscutablement humain ${ }^{59}$ ?

D'autres schémas de structure inerte pourraient être envisagés, sans convaincre davantage: une correspondance «chiasmatique » entre D 1 et D 449, D 2 et D 448, etc., jusqu' à celle entre D 224 et D 226, qui renforcerait le rôle de pivôt du D 225, privé d'équivalent. Ou encore, de chaque côté du diptyque, et en excluant également D 225 pour une question de parité, une correspondance entre D 1 et D 224 d'une part, D 226 et D 449 de l'autre, jusqu'à la correspondance entre $\mathrm{D} 112$ et $\mathrm{D} 113$ dans la première partie, $\mathrm{D} 337$ et $\mathrm{D} 338$ dans la seconde. Ou enfin, un système de reprise qui ferait de D 226 l'homologue de D 1, de D 227 celui de D 2, etc., jusqu'à la fin. Malgré de patientes et acrobatiques recherches, il faut se rendre à l'évidence, ce serait faire trop bon marché du contenu prédicatif précis de chaque dizain que de vouloir à toute force fonder ces parallélismes. Et il n'est même pas sûr que la tentative ne contrevienne pas à la littérarité poétique comme telle ${ }^{60}$.

Aucun "patron » numérologique ne paraît donc satisfaisant, aucun « ordre ", au sens d'une disposition concertée délivrant en elle-même un sens global à l'échelle du recueil, ne semble susceptible d'intégrer toutes les marques d'hétérogénéité, d'irrégularité ou de débordement qu'il présente - voire, tout simplement, de déjouer les risques de contresens sur tel ou tel dizain (et le recueil en fourmille littéralement) en fournissant au lecteur un aiguillage interprétatif suffisamment consistant, c'est-à-dire précis et dépassant la seule association d'idées. Et pourtant, il y a bien un ordre, une régularité extrême et affichée, un rigoureux agencement de la facture; et il y a bien aussi un sens général, puisqu'il y a des constantes prédicatives, au premier rang desquelles l'affirmation jamais démentie d'une posture adoratrice. Faut-il renoncer à concilier les deux versants du problème?

Mieux vaut envisager une solution de type dialectique, qui relierait précisément l'émergence d'un sens à la systématique défaillance d'un ordre. Scève ne s'ingénie peut-être à suggérer les constructions intégratrices qu'afin de montrer que la vérité de son dire poétique tient à leur inefficacité. Le sens implicite serait alors que l'Amour comme tel est désordre, perte de repères, même quand ne reste de certitude que celle d'une hiérarchie vécue et assumée, donc d'un ordre existentiel. Ce faisant, c'est l'entreprise même du canzoniere qui est en jeu, le poète étant toujours débordé, dans l'écriture aussi raisonnée que possible de son expérience, par ce qui perturbe ses facultés. Une telle hypothèse est

59. Voir les remarques de G. Defaux sur l'intertextualité numérique en question et, plus largement, la dimension religieuse de l'œuvre, éd. cit., p. lxxxiv-lxxxvi.

60. Comme le dit avec une certaine irritation D. G. Coleman, « $[\ldots]$ it is dishonest to talk about the macrotextual structure, the numerical patterning, the sequential linking device, or to try and prove anything by "breakdown of structure" rules or by making the number 6 the controlling point of the text » («The Poetic Sensibility of Scève », p. 126-127, dans Pre-Pléiade Poetry, Lexington, French Forum, 1985, p. 125-135). 
d'autant moins fantaisiste que toutes les perplexités qui précèdent se résument à l'hésitation continue entre deux grands systèmes de construction, qui supposent deux dispositions mentales a priori incompatibles et que figurent deux types d'espace: d'un côté la Délie est un temple, de l'autre c'est un labyrinthe.

D'une certaine manière, ce flottement est annoncé dès le premier dizain, comme s'il s'agissait d'y définir les deux types de seuil que le lecteur vient de franchir simultanément: si d'une part Délie est «Constituée Idole » de la vie de l'Amant, qui devient sa "Piteuse hostie » - donc victime sacrificielle compensatoire ${ }^{61}$, avec toutes les connotations religieuses qu'implique la notion -, et donc si la Délie s'affiche comme temple où trône une exigeante divinité, d'autre part, chez l'Amant, «l'Esprit desvie », sort de ses voies normales, s'égare jusqu'à se perdre - ce dont témoigne aussi sa « Raison despourveue » incapable de retrouver ses repères -, dessinant ainsi l'entrée dans un labyrinthe. Le lieu de l'adoration est aussi un lieu de dispersion, si ce n'est de perdition, la grande question étant alors de savoir quel type d'issue l'emporte effectivement.

Avant d'envisager les différences concrètes qu'illustre le texte entre ces deux espaces, il faut rappeler ce que ces derniers ont tout de même en commun: un lieu relativement clos, artificiel ${ }^{62}$, qui enclôt un mystère et suggère un parcours. Celui qui y pénètre ne le fait pas pour satisfaire aux besoins les plus naturels, mais pour y chercher quelque chose ou quelqu'un dont l'importance est soulignée par la complexité de l'espace qui y conduit ${ }^{63}$. Il faut également se souvenir, pour mitiger l'incompatibilité de principe qui vient d'être énoncée, que temple et labyrinthe ont fait bon ménage en certaines occasions: de l'église de San Reparatus ${ }^{64}$ à la basilique San Vitale de Ravenne ${ }^{65}$ et à la cathédrale de Chartres ${ }^{66}$, $1^{\prime}$ architecture chrétienne récupère ad maiorem dei gloriam ce motif éminemment païen qui semble plonger ses racines, bien au-delà de la Crète mi-

61. Le latin classique hostia s'emploie par opposition à victima, « victime offerte en remerciement ». Et comme par un fait exprès, alors qu'il est tout de même question des souffrances d'un Amant et de la cruauté de sa Dame pendant plus de quatre mille vers, Scève n'emploie pas une seule fois le mot "victime », attesté depuis 1495. Certains silences sont éloquents.

62. L'application du terme à des lieux naturels (spéléologiques, anatomiques) est une extension de ce qui concerne d'abord un bâtiment dont il est difficile de sortir.

63. Même les églises les plus dépouillées, les temples romains les plus frustes, présentent une structure, un jeu de proportions, une dispositio. Ce n'est pas Vitruve ni Alberti qui diraient le contraire.

64. Le plus ancien exemple connu (328), à Castellum Tinginatum (aujourd'hui El Asnam, Algérie).

65. Le labyrinthe dessiné sur le sol est ainsi fait que c'est depuis son centre que le visiteur a le meilleur point de vue sur les mosaïques des murs et du plafond en entier, donc accès à l'intégralité du message.

66. Comme tous les autres labyrinthes médiévaux retrouvés ou décrits, il s'agit d'un labyrinthe à trajet unique, sans bifurcation, qui aboutit à coup sûr à un espace central symbolisant le salut du chrétien; la difficulté pour celui-ci n'est pas de faire des choix d'orientation, mais d'endurer les lentes sinuosités décourageantes (à suivre à genoux) pour les hommes de peu de foi. Il est classique d'y voir aussi un substitut mimétique au pélerinage en Terre Sainte. 
noenne, dans l'Égypte des complexes funéraires ${ }^{67}$. Ces deux espaces sont donc à la fois dédiés à une préservation, à une initiation, et à la rencontre de la vie et de la mort. Enfin, sur un tout autre plan, celui de l'écriture, Scève n'emploie ni l'un ni l'autre de ces deux termes dans la Délie - ce qui ne suffit évidemment pas à fonder leur pertinence, mais ce qui est nettement moins gênant que si un seul des deux était employé.

Cela étant dit, la concurrence ou la combinaison de ces deux types d'espace devant se traduire par des différences concrètes au niveau du fonctionnement sémantique de la Délie, il est nécessaire de donner un aperçu synthétique de $l^{\prime}$ une puis des autres ${ }^{68}$, à travers le tableau suivant:

\begin{tabular}{|c|c|c|c|}
\hline & - Critères/Epaces - & Temple & Labyrinthe \\
\hline I & Dimension majeure de l'espace & Verticalité & Horizontalité \\
\hline II & Relation ordre/sens & Sens - ordre & Ordre - sens \\
\hline III & But du parcours & Salut & Survie \\
\hline IV & Attitude requise & Adoration & Enquête/Lutte \\
\hline V & Teneur majeure du discours & Expressivité & Connaissance \\
\hline VI & Statut de l'Aimée & Divinité & Monstre \\
\hline VII & Statut de l'Amant & Fidèle & Adversaire \\
\hline VIII & Emblème-type & $\begin{array}{c}\text { E 3: « La Lampe \& } \\
\text { l'Idole » }\end{array}$ & E 46: «L'Yraigne» \\
\hline IX & Exemplarité & $\begin{array}{c}\text { Immédiate et évi- } \\
\text { dente }\end{array}$ & Problématique \\
\hline
\end{tabular}

Les trois premières lignes de ce tableau n'ont guère besoin d'éclaircissement, puisqu' elles se ramènent à un distinguo fondamental entre la transcendance du temple et l'immanence du labyrinthe. L'« horizontalité » de ce dernier signifie le caractère essentiellement intramondain du parcours qu'il propose, car même dans les cas d'exploitation symbolique (dans l'alchimie, et plus largement l'ésotérisme), la chambre centrale révèle à celui qui y pénètre sa propre vérité intérieure, les circonvolutions de sa quête lui indiquant ironiquement que l'homme

67. Il faut ici rappeler $1^{\circ}$ ) que le plus ancien témoignage occidental sur un labyrinthe réel est celui d'Hérodote, à propos du complexe de "trois mille " pièces bâties sous le roi Amenemhet III à Hawara, dans le Fayoum (XIX ${ }^{\mathrm{e}} \mathrm{s}$. av. J.-C.), d'un intérêt pour lui bien supérieur à celui des pyramides (L'Enquête, II, 148$) ; 2^{\circ}$ ) que cette dénomination s'est faite par référence analogique au labyrinthe mythique de Crète; $3^{\circ}$ ) que néanmoins Dédale, au rapport de Pline (Hist. Nat., XXXVI, 84-85), se serait inspiré du modèle égyptien pour réaliser la commande de Minos; $4^{\circ}$ ) qu'aucune étymologie pleinement convaincante n'a été proposée pour ce mot de «labyrinthe ».

68. De façon évidemment simplificatrice, puisque la variété de leur réalisation peut toujours fournir un contre-exemple. Sera donc ici appelé «temple» tout lieu de culte consacré à une divinité (que sa figure y soit présente ou non), et le modèle du « labyrinthe » sera celui du mythe de Thésée. 
met du temps à se retrouver face à lui-même ${ }^{69}$. Surtout, l'entrée périlleuse et la difficile sortie, comme épreuve formatrice, sont à elles seules suffisantes pour rendre compte de l'existence de l'édifice, qui n'a pas besoin d'une vérité transcendante pour exister. $C^{\prime}$ est ce qu'indique la deuxième ligne, car si dans le temple l'ordonnancement des diverses parties trouve sa justification dans un sens préétabli que le fidèle est censé connaître (motif de la croix en chrétienté, rétrécissement progressif du plafond dans l'ancienne Égypte, etc.), c'est au contraire l'ordre deviné in fine du labyrinthe qui lui donne son sens (sortie identique ou non à l'entrée, existence ou non d'un point central, etc.).

Les quatre suivantes synthétisent les diverses configurations relationnelles qu'installe le texte entre l'Amant et Délie: le recueil fait alterner, parfois à l'intérieur d'un même dizain, une posture de demande et de célébration qui justifie l'appellation d'Aimée pour sa destinataire, donc au moins virtuellement lyrique - et il y a bien une lyrique religieuse -, et une posture d'étude attentive, presque de didactisme, qui va forcément de pair avec le refus d'un joug insupportable, avec un recul critique devant la « doulce ennemie ", pour reprendre un syntagme fréquent. Le véritable « mariage de la carpe et du lapin » est peutêtre là: pour éperdue que soit sa passion, jusqu'au bout l'Amant refuse d'être dupe, tâche de comprendre, d'analyser l'alchimie de ce qu'il ressent - d'où, notamment, les nombreux dizains " psychérologiques » ${ }^{70}$. Cette dimension est du reste clairement assumée par l'Amant, notamment aux Dizains 202 et 218, bons représentants de cette catégorie, et cités ici respectivement:

T'esbahys tu, ô Enfant furieux,

Si diligent la verité je tente?

Et l'esprouvant, me dis tu curieux

A rendre en tout ma pensée contente ${ }^{71}$ ?

Bien que j'acquiere en souffrant la science

De parvenir a choses plus prosperes,

Si n'est ce pas (pourtant) qu'en patience

$\mathrm{J}^{\prime}$ exerce en moy ces deux uterins freres ${ }^{72}$

Les limites assignées à la présente réflexion ne permettent pas de vérifier très longuement la validité de cette combinaison problématique entre les deux structures mentales. Cependant, à la fois pour permettre sa mise à l'épreuve de manière pragmatique et pour l'esquisser ici sur un plan systématique, qu'il

69. «Le labyrinthe représente le voyage psychique et spirituel que l'homme doit accomplir à l'intérieur de lui-même, à travers les épreuves et tous les motifs d'égarement, afin de trouver son propre centre, ou en d'autres termes, l'image de son Soi. Alors, le cœur du labyrinthe est souvent vide - de sorte que le centre est à la fois la plénitude et le vide. " (Encyclopédie des symboles, M. Cazenave éd., Paris, L.G.F., 1996, ad loc.)

70. Néologisme transparent forgé pour les besoins de la cause dans le travail de doctorat signalé supra.

71. V. 1-4.

72. V. 7-10. Les frères en question sont «Amour ardent, \& Cupido bandé », enfants jumeaux de Vénus cités au dizain précédent. 
soit maintenant permis de tenter une conjonction entre les trois grands types de discours qui nourrissent le recueil et le paradigme proposé, conjonction qui sera illustrée à chaque fois par un exemple.

La Délie, en effet, présente trois grands types d'énoncés: un premier ensemble formé de vœux, d'exhortations, de requêtes plus ou moins mêlées de plaintes (avec priorité au futur) ; un ensemble de récits, anecdotes et paraboles, accompagnés ou non d'une « leçon » (avec priorité au passé); un ensemble de diagnostics (avec priorité au présent). Caractéristique du premier groupe, le D 265 s'achève sur une requête de présence auprès de l'Amant:

Vien, Dame, vien: Asses as esclercy

Ces champs heureux, ou a present sejourne

Ton Orient, \& en la Ville icy

Jamais, sans toy, a mes yeulx ne s'ajourne ${ }^{73}$.

Il s'agit bien d'une prière, d'une demande de grâce: par sa présence effective, Délie fera que le jour vécu par l'Amant sera digne de ce nom, et le versant adorateur joue ici à plein. Mais le rapprochement avec le dizain précédent fait éclater une contradiction, puisqu'il affirme sans ambages que la mémoire affective de l'Amant est si intense qu'elle dépasse l'importance de la présence réelle de l'Aimée:

Tes fiers desdaingz, toute ta froide essence,

Ne feront point, me nyant ta presence,

$\mathrm{Qu}$ 'en mon penser audacieux ne vive,

Qui, maulgré Mort, \& maulgré toute absence,

Te represente a moy trop plus, que vive $\mathrm{e}^{74}$.

Pour le coup, le lecteur passe d'une case à l'autre du labyrinthe, avec la succession de ces deux assertions difficilement compatibles - et ces palinodies sont loin d'être isolées. Le cas échéant, d'ailleurs, c'est un même dizain qui les englobe ${ }^{75}$.

Le fameux «dizain des joutes » (D 286) illustre quant à lui cette double structure mentale dans l'ordre narratif: du côté du «temple », le pari raté de l'Amant sur l'identité du vainqueur renseigne implicitement sur les contraintes du culte envers Délie, qui peut s'adosser à la piété chrétienne: elle semble favoriser l'humilité, puisque c'est « le hault » qui tombe à l'eau; mais, du côté du labyrinthe, la scène évoque fort ces devinettes perverses dont est parsemé le tortueux parcours de l'aventurier, et qui lui sont une collection d'indices quant à la personnalité du monstre à affronter (car il s'agit de « victoyre avoir » ${ }^{76}$ sur

73. V. $7-10$

74. V. 6-10.

75. «Je m'en absente \& tant, \& tant de foys,/Qu'en la voyant je la me cuyde absente:/Et si ne puis bonnement toutesfoys,/Que, moy absent, elle ne soit presente» (D 215).

76. V. 4. C'est «Amour, qui vint les joustes veoir », qui lui propose cette gageure, non sans ironie («Eslis (le mieulx, que tu pourras sçavoir)/L'un de ceulx cy »...). 
la Dame, pas simplement de lui complaire). Étant donné les enjeux, ce n'est pas là que métaphore: dans le premier cas, il y a de fortes chances que le parcours finisse par être éclairant, le "merite » herméneutique de l'Amant se trouvant récompensé par la communauté d'immortalité du « Genevre » final, et le dizain figure une étape d'échec instructif; dans le second, l'Amant est une fois de plus circonvenu dans ses tentatives de constituer une axiomatique fiable, et l'issue n'est pas près d'être trouvée; pis encore, la «victoyre », ou une certaine sorte de succès, lui devient interdite.

Pour ce qui concerne enfin les diagnostics, le D 376 fournit une substantielle illustration de ce flottement: d'une part, en effet, l'Amant y rappelle l'étroite dépendance de son être envers Délie, à travers la reprise de la métaphore du corps et de l'ombre ("Tu es le Corps, Dame, \& je suis ton umbre», v. 1); mu « par povoir de [s] a haulte excellence » (v. 5), il finit par sentir «trop inhumainement" (v. 9) leurs «sainctz vouloirs estre ensemble discords » (v. 10). L'indignité du fidèle en tant que second ne fait que corroborer le service qu'il rend presque malgré lui à son idole. Mais, curieusement, ce dispositif élémentaire est perturbé par la résurgence mécanique d'un autre dizain, qui associait étroitement Délie à Hécate ${ }^{77}$, car Délie le fait ici « mouvoir, non comme Hecate l'Umbre,/Par ennuieuse, \& grande violence », mais précisément par son « excellence». À ce déplacement de l'image ou du statut de l'Aimée s'ajoute un détail lexical intéressant qui renforce le contraste avec l'infernale déesse: Délie mène $l^{\prime}$ Amant « au doulx contournement/De tous [s] es faictz » (vv. 6-7) ${ }^{78}$, et cependant «plus soubdainement » que l'ombre ne suit le corps. Nul besoin de forcer ce terme de « contournement » pour saisir toute la dangereuse sensualité que dégage le parcours labyrinthique d'un Amant littéralement fasciné, hypnotisé par son guide fatal, à grand-peine entendu, à grand regret suivi. Si les «sainctz vouloirs » sont « ensemble discords », c'est donc peut-être que Délie mène l'Amant à sa perte pour son (plus hault) bien, alors qu' elle-même, en tant que fin mot de la quête, énigme qui s'ignore en tant que telle, ne perd rien dans le cours de l'enquête.

Les exemples pourraient être multipliés, sans qu'il faille déployer des trésors de subtilité. Et pour une raison aussi simple que radicale, sur laquelle il est maintenant possible d'esquisser une conclusion: dans chacun de ses dizains, dans chaque neuvaine, comme à l'échelle du recueil dans son ensemble, ou même quelle que soit la subdivision technique ou thématique considérée, Scève permet au lecteur de déceler aussi bien les éléments de constitution d'un temple poétique dédié à l'Aimée que les couloirs et les salles d'un labyrinthe où se joue la survie de l'Amant - dans les deux cas, une « si haulte architecture » dont il fait seul les frais à mesure qu'il la réalise. Là est son édifice - peut-être incons-

77. « Comme Hecaté tu me feras errer/Et vif, \& mort cent ans parmy les Umbres » (D 22, vv. 1-2).

78. Ce que F. Joukovsky est la seule à gloser correctement « en suivant en souplesse (doulx: sans violence; cf. v. 5) la trace sinueuse de tes actions » (éd. cit., p. 353.). 
cient, peut-être imparfaitement maîtrisé, peut-être work in progress inavoué -, là est son intérêt, et là est sa limite. Car dans l'économie de l'œuvre, chaque dizain l'égare parce qu'il le renseigne: arrivant toujours à une leçon sur lui et sur lui seul, il se rend impossible un culte naïf; et réciproquement chaque dizain le renseigne précisément parce qu'il l'égare: en lui démontrant sa confusion, des ordres, des valeurs, des facultés, il s'indique à lui-même un nécessaire et sempiternel sursaut. Rien d'étonnant, dès lors, à ce que soient conjointement et simultanément reconnues deux caractéristiques du recueil qui rendent compte de l'ensemble de ces relations entre l'ordre et le sens: raffinement technique, jusque dans le lyrisme, obscurité, jusque dans l'explication. Cela n'est concevable concrètement que si le poète fait les frais d'une double démarche dont il n'avait pas mesuré l'incompatibilité dès le départ: jouer à un jeu dont on définit les règles en même temps qu'on s'y engage, et donc sans en deviner l'issue.

Clivage inouï de Scève exorcisant sa posture d'Amant: entreprenant de construire un temple magnifique de symétrie, dédié à l'idole qu'il a lui-même façonnée, il s'enfonce et s'enferme lentement, dizain après dizain, dans un labyrinthe truffé de décrochements et de distracteurs auquel sa contrainte initiale lui interdit de donner la moindre issue: 1 'introuvable chambre centrale passe entre les D 224 et D 225, comme entre les emblèmes 25 et 26, dans la béance entre la moitié par défaut et la moitié par excès ${ }^{79}$.

Cumul inouï des rôles dans cette aventure singulière: non seulement celui du grand prêtre, du fidèle de base et de l'architecte du temple, mais encore celui de Thésée bien décidé à triompher d'un monstre, celui du monstre lui-même déchiré entre animalité des pulsions et humanité de l'esprit, celui d'Ariane qui donne le fil, et celui de Dédale qui ne parvient qu'à grand-peine à ressortir vivant de l'univers de leurres qu'il a conçu. C'est dans sa lutte raisonnée contre son propre émiettement que le Moi se subdivise et se réalise en une poussière d'épigrammes, rendant ipso facto impossible sa persistance dans quelque paratexte que ce soit.

\section{Xavier BONNIER Université de Toulouse-Le Mirail}

79. Corollaire lexicologique: le mot même de « centre », dans deux au moins de ses quatre occurrences (D 165 et D 228), est loin d'être clair. 